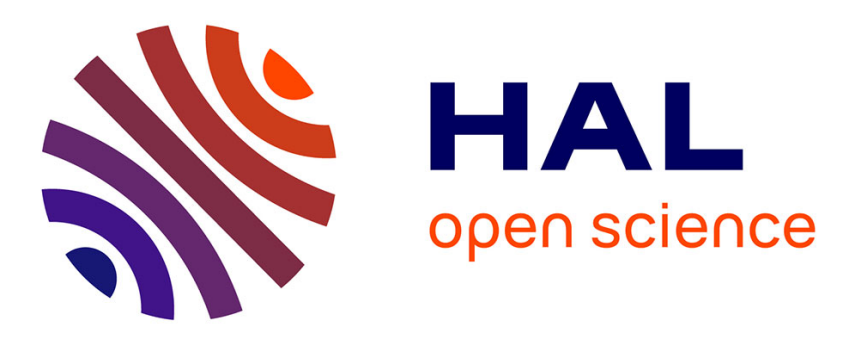

\title{
Efficient Self-optimization of Neighbour Cell Lists in Macrocellular Networks
}

\author{
van Minh Nguyen, Holger Claussen
}

\section{To cite this version:}

van Minh Nguyen, Holger Claussen. Efficient Self-optimization of Neighbour Cell Lists in Macrocellular Networks. PIMRC 2010 - IEEE 21st International Symposium on Personal Indoor and Mobile Radio Communications, Sep 2010, Istambul, Turkey. pp.1923 - 1928, 10.1109/PIMRC.2010.5671609 . hal-00629694

\section{HAL Id: hal-00629694 https://hal.science/hal-00629694}

Submitted on 7 Oct 2011

HAL is a multi-disciplinary open access archive for the deposit and dissemination of scientific research documents, whether they are published or not. The documents may come from teaching and research institutions in France or abroad, or from public or private research centers.
L'archive ouverte pluridisciplinaire HAL, est destinée au dépôt et à la diffusion de documents scientifiques de niveau recherche, publiés ou non, émanant des établissements d'enseignement et de recherche français ou étrangers, des laboratoires publics ou privés. 


\section{Efficient Self-optimization of Neighbour Cell Lists in Macrocellular Networks}

\author{
Van Minh Nguyen \\ Bell Labs, Alcatel-Lucent, 91620 Nozay, France \\ van_minh.nguyen@alcatel-lucent.com
}

\author{
Holger Claussen \\ Bell Labs, Alcatel-Lucent, Dublin 15, Ireland \\ holger.claussen@alcatel-lucent.com
}

\begin{abstract}
The neighbour cell list (NCL) in cellular networks has an important impact on the number of dropped calls and is traditionally optimized manually with the help of planning tools. In this paper, a method for automatically optimizing a NCL is presented, which consists of an initialization using a selfconfiguration phase, followed by a self-optimization phase that further refines the NCL based on measurements provided by mobile stations during the network operation. The performance of the proposed methods is evaluated for different user speeds and different NCL sizes. Besides, the convergence speed of the proposed self-optimization method is evaluated. It is shown that when about 6000 measurements are reported by mobile stations, the proposed self-optimization method attains a stable maximum performance about $99 \%$ of success rate.
\end{abstract}

Index Terms-neighbour cell list, self-optimization, selfconfiguration, cellular networks

\section{INTRODUCTION}

Handover is one of the most critical issues in cellular networks. It enables connection continuity for mobiles during move, while allowing the efficient use of resources (e.g., time and frequency reuse between cells). Most of today's cellular standards use mobile-assisted handover in which the mobile measures the pilot channel signal quality of neighbour cells and reports the measurement result to the network. If the signal quality from a neighbour cell is better than that of the serving cell by a handover margin, the network initiates a handover to that cell.

Today, commercial cellular networks use a neighbour cell list (NCL) to control the neighbour cell measurements by mobiles. In order to measure neighbour cells, the mobile needs to be provided with information on the pilot channels of potential neighbour cells (e.g., cell synchronization information, pilot channel frequency). If this information is not provided by the network, the mobiles have to spend a longer time to acquire it via scanning through all possibilities. Therefore, a list of neighbour cells as handover candidates is configured for each cell. This list contains information of the selected handover candidates and is sent to all mobiles connected to the cell. The mobiles then only need to monitor the pilot signal quality of the cells included the NCL of the serving cell.

The neighbour cell list has an important influence on the mobility performance. It must contain a sufficiently large number of potential neighbour cells to ensure that any mobile in the serving cell can find at least one handover target when its own signal deteriorates. However, as the mobile measurement capacity is limited, a long list can result in delays in finding a suitable handover target. These delays may cause call drops when the user moves at high speed.

While it might appear easy to configure a neighbour cell list by simply looking at the cell topology and selecting all cells overlapping with the cell of interest, it is not as straightforward in reality. This is mainly due to the fact that the real radio coverage is unknown, since it depends largely on the environment which can only be modelled to some degree in radio simulations. For instance, the presence of unpredicted buildings, trees, and moving objects will have effects on the coverage and may create coverage holes not predicted by the model. In addition, changes in the environment due to for example newly constructed buildings cannot easily be taken into account. Thus, an accurate prediction of the cell coverage is hard and also might not be valid anymore after some time.

In recent practice, the NCL is still manually configured at the beginning of the network deployment by means of planning tools, and is manually updated when new cells are installed. During this manual configuration the cell coverage and the neighbour relation are predicted using static information such base station locations, antenna patterns, and received signal maps (obtained through driver-tests). Due to the sensibility and the dynamic of the radio propagation condition, these static predictions of the cell coverage are more or less inaccurate, and cannot take changes in the radio conditions into account. More details of the drawbacks of the manual NCL configuration can be found in [1], [2].

Therefore, the manual configuration and optimization of the neighbour cell list is a non-trivial problem that requires efforts for the network operator, resulting in considerable operational expenses. In [1], Olofsson et al. explained how a selfoptimization approach is able to overcome these difficulties. In addition, the Next Generation Mobile Network (NGMN) alliance recently specified neighbour cell list self-optimization as a required use case [3], and described its benefits for the network operator [4].

A key element of the neighbour cell list configuration is reliable information on the cell coverage. In a real network, the best way to obtain accurate coverage information is to continuously perform measurements of the radio signals. The most efficient way to achieve this is to let the network entities such as mobile stations and base stations perform measurements during operation. In this paper we propose a 
method which uses live measurements to self-configure the initial neighbour cell list, and to continuously self-optimize the neighbour cell list of a cell during operation. The paper provides the following contributions:

- A complete optimization procedure which self-configures an initial NCL and continuously self-optimizes the NCL.

- An efficient method which does not generate additional overheads by using the mobile-reported measurement available by the standard requirement.

- A definition of the optimal neighbour cell list.

- Efficient algorithms which avoid static constraints and compute the NCL from live measurements. This is important as it frees the operator from a tricky manual collection of the network configuration, and allows the NCL to be quickly adapted to the changes in the network and its environment.

The rest of this paper is organized as follows. In Section II, related work in this area is discussed. Section III describes the concept of the proposed method. The self-configuration method used for initialization is described in Section IV. In Section V, the ongoing self-optimization process is described. Here, during the network operation, connected mobiles measure neighbour cell pilot signals and report results to the serving base station. Then the NCL is optimized such that it maximizes the probability of finding an appropriate handover target. Section VI presents the performance evaluation using simulations based on a real network deployment scenario. The paper is concluded in Section VII.

\section{RELATED WORK}

The general concept of dynamic neighbour cell list planning in [1], [5] is one of the first attempts in the area of neighbour cell list self-optimization. In this concept, a neighbour cell is dynamically added to the NCL if the percentage of handovers from the serving cell to this neighbour cell is above a predefined threshold. Now and then some test frequencies are added to the neighbour frequency list to allow mobiles measuring cells which are not currently in the NCL. This aims at discovering potential good neighbours.

Following the motivation introduced in [1], [5], Guerzoni et al. [6] proposed an automatic optimization algorithm. This algorithm mainly consists of identifying and ranking missing neighbours. The neighbours are identified analytically, and then ranked based on the base station coordinates and the antenna direction. In parallel, the cells currently in the NCL are assessed using key indicators such as handover success rate and received signal quality. After that, good cells from the pool of missing cells are added to the NCL while bad cells in the NCL are removed.

Realizing that the method proposed in [6] may produce a very large pool of potential missing neighbours, and that the statistical confidence on the parameters used for ranking the cells may not be optimal, Soldani and Ore [7] proposed an enhanced procedure for UTRA FDD networks. Here, detected set reporting is used to identify missing neighbours, then thresholds are used as a coach filter to limit the pool list.
The objective here is to efficiently identify and filter missing cells, but not to compute an optimal NCL.

Further work by Parodi et al. [8] focused on automatically creating a NCL for newly installed cells. Slightly similar to [7], cells located in a given range from the new cell are filtered out as potential neighbours. Then, based on prediction models, each cell in the pool is checked for overlap with the new cell. A number of the best neighbours are finally selected for the NCL. In addition, a signalling implementation for LTE networks is proposed to allow a newly installed cell to detect its neighbours.

Three self-configuration methods for WiMAX networks are proposed in [9] and are very similar to those discussed in [8].

In [2], [10], live measurements are considered for the neighbour relation table optimization and physical cell identifier (PCI) conflict detection in LTE.

The above studies contributed valuable concepts for the self-optimization of the NCL. However, when realizing the proposed concepts, they are mostly based on static information and static thresholds which limit the performance and the ability to adapt to dynamic changes in the radio environment.

\section{CONCEPT}

The concept of the proposed NCL self-optimization method is conceived on the basis of the following questions:

- What is the definition of an optimal NCL?

- What measurement statistics are needed and how can they be obtained?

- How can potential neighbours be discovered that are not currently included in the neighbour cell list?

The objective of the neighbour cell list is to assist mobile stations in finding a suitable handover target. A neighbour cell is considered as a suitable handover target for a mobile if its pilot signal quality received by the mobile is higher than a minimum requirement $P_{\min }$, for example $P_{\min , \mathrm{E}_{\mathrm{c}} / \mathrm{I}_{0}}=-20 \mathrm{~dB}$ in WCDMA [11]. In addition, the information obtained by measurements of the pilot signals of neighbour cells must be of good statistical confidence. The shorter the neighbour cell list is, the more frequent the neighbour cells can be measured, which results in a higher confidence in the measurements. In reality, the maximum size of the neighbour cell list is limited by the standard, such as to 32 cells as specified by $3 \mathrm{GPP}$. From the above considerations, the optimal neighbour cell list is defined as the one that has the least cells within the standardized allowable number (e.g., 32), and maximizes the probability of finding a handover target. Here the probability of finding a handover target is defined as the probability that at any location in the serving cell the mobile finds at least one suitable handover target from the neighbour cell list.

The measurement statistics needed are the indicators that are used for the handover target selection such as SINR (signal-to-interference-plus-noise ratio) in GSM, and $\mathrm{E}_{\mathrm{c}} / \mathrm{I}_{0}$ in WCDMA. Since this information is only available when the network is operational but not in the planning phase, the proposed optimization method is composed of two phases: 


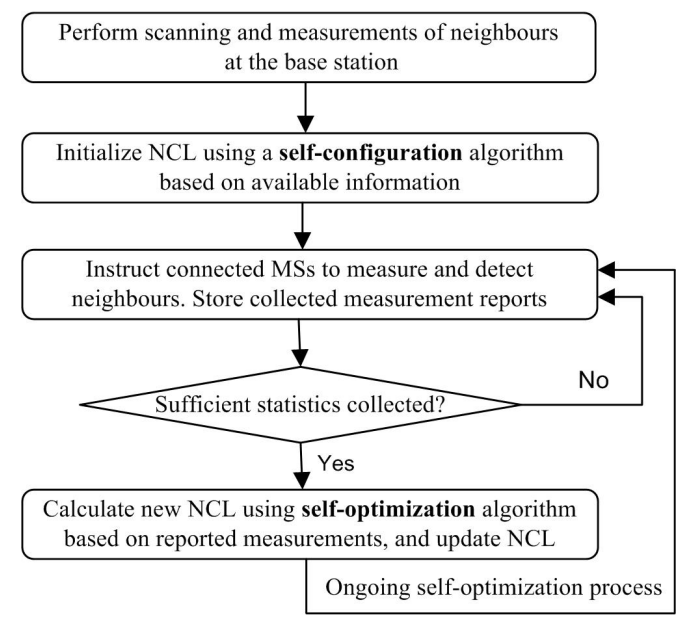

Fig. 1. Principle of neighbour cell list self-optimization

self-configuration for the network initialization, and ongoing self-optimization during the network operation, c.f., Fig. 1.

When the network is newly deployed, neighbours are identified and measured by each base station on the downlink band. The neighbour cell list of each cell is then initialized using a self-configuration algorithm based on the collected measurements. This phase is also applied to self-configure a initial neighbour cell list of a new cell that is installed when the network is already operating.

During the network operation, connected mobiles are instructed to perform measurements using standardized scanning procedures, and report results to the serving base station. This phase of collecting measurement statistics also deals with the cell discovery. In broadband networks such as WCDMA, mobiles are capable of detecting and reporting cells even when they are currently not included in the neighbour cell list ${ }^{1}$ [11], [12]. For multi-carriers networks like GSM, the cell discovery can be done by using the concept proposed in [5].

The collected measurement statistics are then used by a self-optimization algorithm implemented in the base station to continuously refine the neighbour cell list. The update of the current neighbour cell list with the self-configured or selfoptimized neighbour cell list can be totally automatic, or with the intervention of the operator to allow the introduction of additional specific policies (for example to remove prohibitive cells is not performed fully autonomously).

\section{Neighbour Cell List Self-Configuration}

Just after the network planning phase, no measurement from mobiles are available, neighbour cells are identified based on measurements performed by each base station. This is done by using the existing sectorized antenna of the serving base station to scan the downlink band and measure identified cells. Then a predefined number of neighbours with the best received pilot quality are included in the neighbour cell list.

\footnotetext{
${ }^{1}$ This ability does not weaken the importance of the NCL since, as mentioned in the introduction, the mobiles need longer time to measure an unidentified neighbour cell.
}

The serving base station's antenna gain supports the measurements of neighbour cells in the direction of the sector coverage, and thereby helps to detect neighbour cells which overlap with the sectorized serving cell.

This approach does not require the operator to provision information on neighbour cells, and thereby reduces the deployment costs. However, its disadvantage is that the neighbours pilot signal quality can not be measured in the whole serving cell coverage area. This is why further measurements by the mobiles are required. These measurements become available during operation and are used in the ongoing self-optimization process described next.

\section{Neighbour Cell List Self-Optimization}

This section details the measurement statistics retrieval and the self-optimization algorithm.

\section{A. Neighbour Measurement Statistics Retrieval}

During the scanning, the connected mobiles measure the pilot signal of neighbours currently in the neighbour cell list. In parallel, potential neighbours are detected and measured by the mobiles according to the principle explained in Section III. This means that the mobiles perform measurements of neighbours that are either detected during the scanning procedure, or are identified during scanning of test frequencies. The mobiles then send measurement reports to the serving base station.

The serving base station stores a measurement statistics table. The table columns are neighbour cells identifiers, and the table rows are measurement reports sent by mobiles. The value at row $i$ and column $j$ represents the pilot signal quality of cell $j$ received by a mobile reporting the measurement $i$.

Whenever a measurement report is received, a new row is added. If this measurement report contains cells that are not currently present in the table columns, one new column is added for each new reported cell. After that, for the recently added row, columns corresponding to the reported cells are filled with the corresponding reported values.

Each row of the statistics table reflects the coverage of neighbour cells at the reported measured location. Any mobile may send several measurement reports during a scanning procedure, and these may correspond to different measured locations in the serving cell (due to user mobility). Therefore, only the measurement reports are relevant while the identifier of the mobiles performing the measurements is not stored.

Besides, old measurements may be not valid anymore due to potential changes in the radio environment. Therefore, at some point, older rows can be removed from the statistics table.

\section{B. Self-Optimization Algorithm}

The proposed self-optimization algorithm is presented in Algorithm 1. The objective of the algorithm is to select neighbour cells such that the resulting neighbour cell list maximizes the number of locations at which there is at least one suitable target cell from the neighbour cell list available.

The input includes the statistics table, the minimum requirement of the pilot signal quality $P_{\min }$, and the maximum 


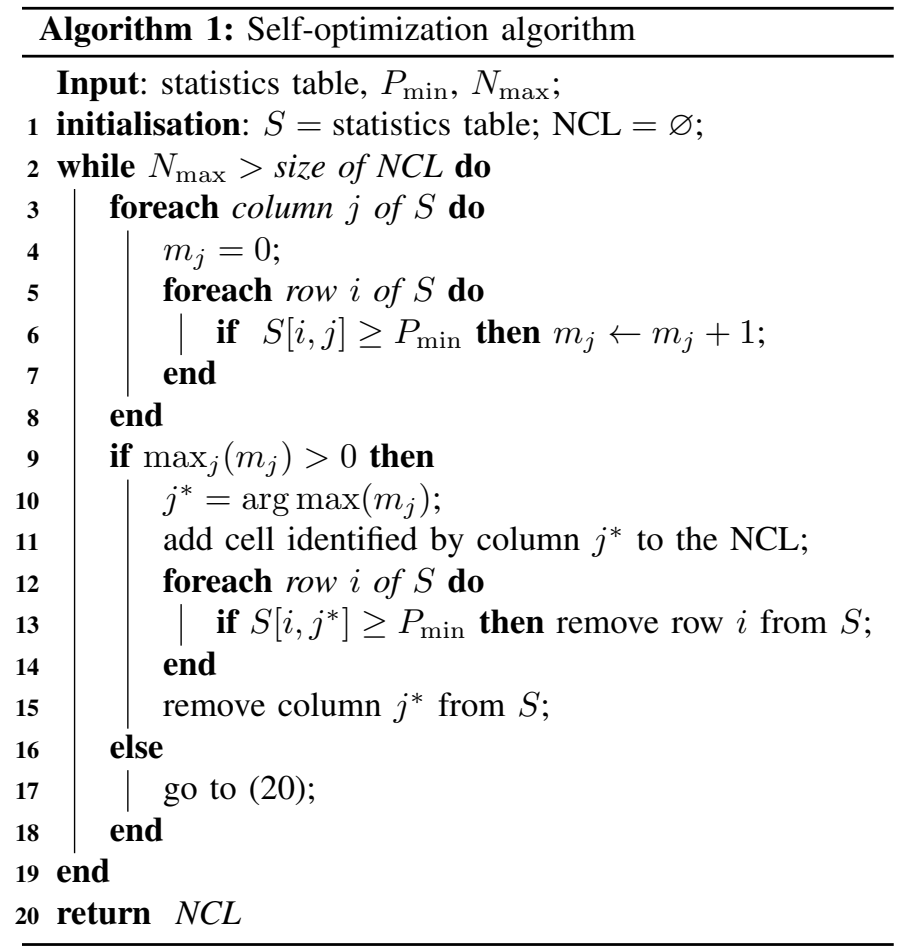

allowable size $N_{\max }$. The "for" loop in lines 3-8 cycles through the number of locations (i.e., number of table rows), $m_{j}$, at which neighbour cell $j$ is a suitable handover target.

Between the remaining neighbour cells in table $S$, the cell corresponding to column $j^{*}$ (line 10) is the best one that maximizes the number of locations covered by the neighbour cell list, and thus is added to the neighbour cell list (line 11). The case of $\max _{j}\left(m_{j}\right)=0$ ("else" in line 16) means that all the remaining neighbour cells in table $S$ do not cover any location of the serving cell with acceptable signal quality. Adding any of these cells will not improve the neighbour cell list. In this case, the resulted neighbour cell list is the best one that maximizes the probability of finding handover target, and so the algorithm is terminated (lines 17 and 20).

When the neighbour cell identified by column $j^{*}$ is included in the NCL (line 11), this column $j^{*}$ and the corresponding rows can be removed (by line 15, and 13, resp.). The cell identified by column $j^{*}$ ensures that the mobiles will find at least one suitable handover target at the locations (i.e., rows) that satisfy the "if" condition in line 13. As the objective is to find the shortest neighbour cell list that covers a maximum number of locations, only the locations that are still not covered by the current NCL are considered in the next rounds.

\section{Vi. Simulation}

This section provides the system model and assumptions, and the simulation results.

\section{A. Simulation Scenario}

A cellular network deployment in Phoenix in the United States was used for the simulation, from which cell locations and antenna orientations were extracted. The size of the simulated scenario is $16 \mathrm{~km} \times 16 \mathrm{~km}$ and includes 120 macrocell sectors. The propagation path loss for a typically urban environment is modelled as

$$
L_{\mathrm{p}[\mathrm{dB}]}(d)=28+35 \log _{10} d,
$$

where $d$ is the distance from the base station in metres. Shadow fading $L_{\mathrm{s}[\mathrm{dB}]}$ in $\mathrm{dB}$ scale is modelled as random process with normal distribution with $8 \mathrm{~dB}$ standard deviation and a correlation of 0.5 between different sites.

It is assumed that the total transmit power for each base station is $P_{\mathrm{tx}}=20 \mathrm{~W}$ per sector, where $10 \%$ of this power is allocated to a pilot channel $P_{\text {tx,pilot }}$. Each macrocell transmits via a sectorized antenna with a gain pattern of

$$
G_{[\mathrm{dB}]}(\theta)=G_{\max }-\min \left(12 \frac{\theta^{2}}{\beta}, G_{s}\right), \quad-\pi<\theta \leq \pi,
$$

where $\beta=70 \pi / 180$ is the angle where the gain pattern is 3 $\mathrm{dB}$ down from peak, the maximum gain is $G_{\max }=16 \mathrm{~dB}$, and $G_{s}=20 \mathrm{~dB}$.

For any location in the considered scenario the received power from a base station $j$ can be calculated based on (1) as

$$
P_{\mathrm{rx}[\mathrm{dBm}]}=P_{\mathrm{tx}[\mathrm{dBm}]}+G_{[\mathrm{dB}]}(\theta)-L_{\mathrm{p}[\mathrm{dB}]}(d)-L_{\mathrm{s}[\mathrm{dB}]} .
$$

The receiver noise power $N_{0}$ is modelled as

$$
N_{0[\mathrm{~dB}]}=10 \log _{10}\left(k T \times N_{f} \times W\right),
$$

where the effective noise bandwidth $W=3.84 \times 10^{6} \mathrm{~Hz}$, $k T=1.3804 \times 10^{-23} \times 290 \mathrm{~W} / \mathrm{Hz}$, and $N_{f[\mathrm{~dB}]}=7 \mathrm{~dB}$ referring to the noise figure at the UE.

For each location in the scenario, an $\mathrm{E}_{\mathrm{c}} / \mathrm{I}_{0}$ can be calculated. The pilot $\mathrm{E}_{\mathrm{c}} / \mathrm{I}_{0}$ is defined in $3 \mathrm{GPP}$ as the ratio of the received energy per pseudo-noise chip for the $\mathrm{CPICH}$ to the total received power spectral density at the UE antenna connector. It can be calculated for a UE connected to the $m$ th cell as

$$
\mathrm{CPICH} \mathrm{E} \mathrm{E}_{\mathrm{c}} / \mathrm{I}_{0_{i}}=\frac{P_{\mathrm{rx}, \text { pilot }, i}}{N_{0}+\sum_{j} P_{\mathrm{rx}, j}},
$$

where $P_{\mathrm{rx}, \text { pilot }, i}$ denotes the pilot power received from cell $i, P_{\mathrm{rx}, j}$ is the total received power of cell $j$ including data and pilot. The pilot minimum requirement for the $\mathrm{UE}$ is $\mathrm{CPICH} \mathrm{E}_{\mathrm{c}} / \mathrm{I}_{0} \geq-20 \mathrm{~dB}$ [11]. In the scenario considered, this requirement results in a nearly $100 \%$ coverage of the area. Fig. 2(a) shows an example of the highest received pilot $\mathrm{E}_{\mathrm{c}} / \mathrm{I}_{0}$, and Fig. 2(b) shows the corresponding coverage regions based on received pilot $\mathrm{E}_{\mathrm{c}} / \mathrm{I}_{0}$ for the sectors within the simulated area. It is assumed that the UE is capable of measuring $\mathrm{CPICH}$ of 8 identified cells (i.e., cells in the NCL) within $200 \mathrm{~ms}$ [11].

For the NCL performance evaluation, mobiles are randomly placed in the simulated area, and move in the network. Each mobile moves at a given speed and changes its direction after each simulated time step (see, e.g., [13], [14]). User speeds between $5 \mathrm{~m} / \mathrm{s}$ and $50 \mathrm{~m} / \mathrm{s}$ are considered. If the signal quality received from the serving cell falls below the scanning trigger threshold, the mobile starts scanning cells comprised in the 


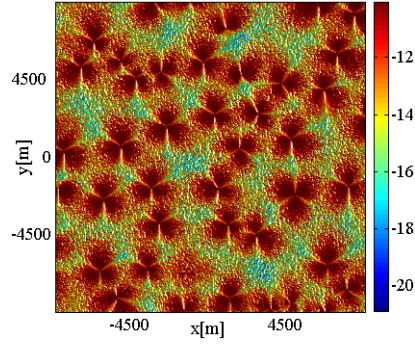

(a) Received $\mathrm{CPICH} \mathrm{E}_{\mathrm{c}} / \mathrm{I}_{0}$ in $\mathrm{dB}$

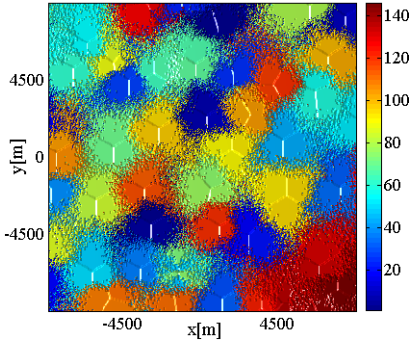

(b) Cell coverage with shadowing
Fig. 2. Simulation scenario

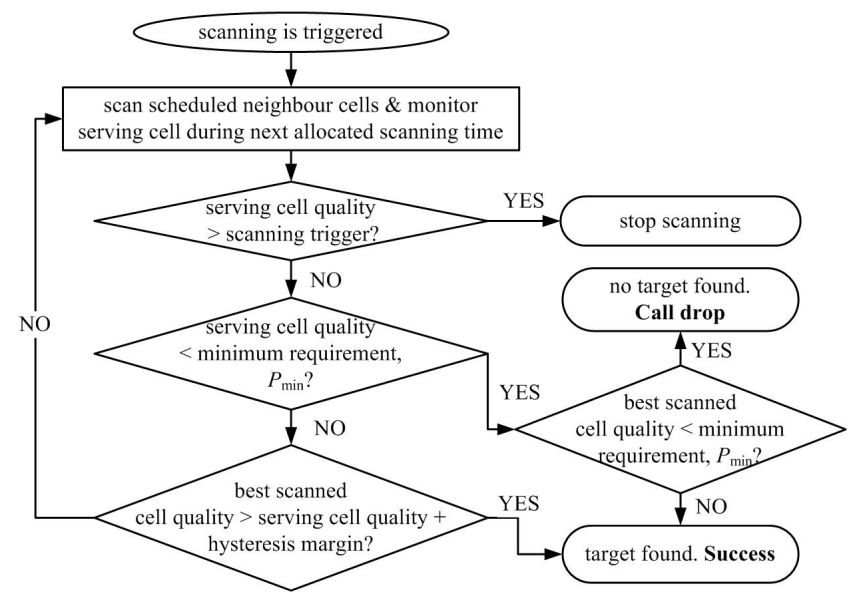

Fig. 3. Studied handover target decision process

NCL. The scanning with handover target selection process is shown in Fig. 3. The handover margin was set to $2 \mathrm{~dB}$. The quality of the NCL is assessed by the handover target searching success rate defined as ratio of the number of mobiles finding a suitable handover target to the total number of mobiles finding a suitable handover target plus the number of mobile which did not find a suitable handover target (resulting in a call drop):

$$
\text { success rate }=\frac{\text { number of Successes }}{\text { number of Successes }+ \text { Calldrops }} \text {. }
$$

Note that after finding a handover target, the mobile enters into the handover execution phase whose outcome depends on the handover algorithm but is not impacted by the NCL. Therefore, only the handover target searching phase is used to assess the NCL quality, the handover execution phase is not taken into the NCL performance evaluation.

\section{B. Performance}

One example of NCL optimization and handover target searching is shown in Fig. 4 for the serving cell 3. Fig. 4(a) shows the results directly after initialization with a NCL computed by the self-configuration algorithm, and Fig. 4(b) shows the results with the NCL that is computed by the selfoptimization algorithm. The optimized NCL has 21 cells. For comparison, a cell list size of 21 was also used for the selfconfiguration algorithm. In Fig. 4, cells comprised in the NCLs

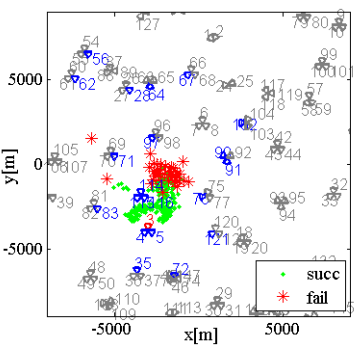

(a) succ rate $=85 \%$ with selfconfigured NCL

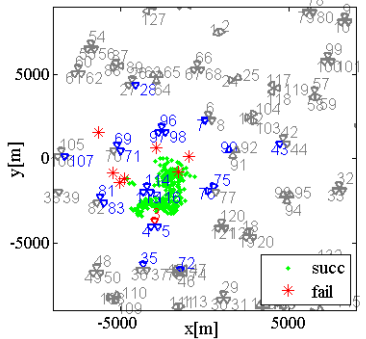

Fig. 4. Examples of scanning from serving cell 3. User speed $v=20 \mathrm{~m} / \mathrm{s}$

are blue-coloured. Comparing Fig. 4(a) and 4(b), the selfoptimization method can improve the success rate of $85 \%$ from the self-configuration to $97.3 \%$. It is shown that three cells 7, 96 and 98 make the difference between the two NCLs. By using mobile-reported measurements, the self-optimization algorithm included these three cells, and thereby eliminates call drops close to these neighbour cells.

For more general results, the handover target searching with NCLs to be evaluated is simulated in 20 serving cells, and the average success rate of each simulated NCL is computed. The self-configuration method is compared to two other methods. The first one is a distance-based method that configures a NCL by selecting a number of the closest neighbour cells. And the second one is similar to the proposed self-configuration method except that the measurements are performed using a separate antenna with uniform gain pattern. These benchmarking methods are marked in Fig. 5(a) \& 5(b) as "distancebased" and "uniform antenna gain", respectively. Note that, as discussed in Section II, self-optimization concepts in the literature are not realized with live measurements like the proposed approach, but with static configuration information. Therefore, no directly comparable benchmark is available. Instead, the self-optimization method is compared with the best self-configuration method for its performance.

Fig. 5(a) shows the performance with different user speeds $v$. It is shown that with a scanning speed of 8 cells $/ 200 \mathrm{~ms}$ as in WCDMA, the user speed does not have much impact on the success rate. It is also shown that the proposed self-configuration method is better than two benchmarking methods, and the self-optimization method performs best.

Fig. 5(b) shows the performance with different list sizes. We see that all the NCLs give better performance for longer lists. This is thanks to the relatively high scanning speed in WCDMA. In some case where scanning speed is low, if the NCL is too long the connection between the mobile and the serving may be lost before finding a suitable handover target. It is also clear from Fig. 5(b) that the proposed self-configuration method is better than two benchmarking methods, and the selfoptimization again performs best. It is shown that the success rates flatten out at about 10 neighbours. From this point on, the performance of the self-optimized NCL approaches a success rate close to $100 \%$, while the configured NCLs have lower 


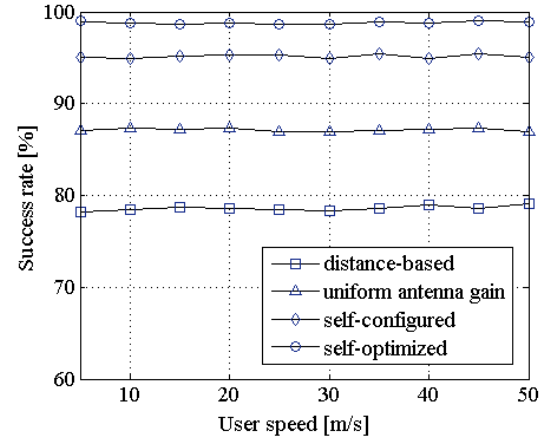

(a) under different user speeds

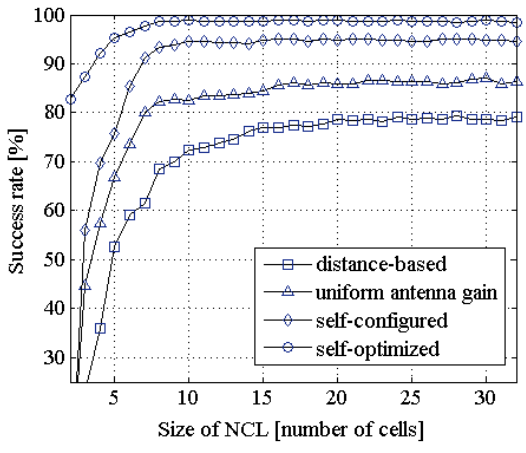

(b) under different list sizes: $v=20 \mathrm{~m} / \mathrm{s}$

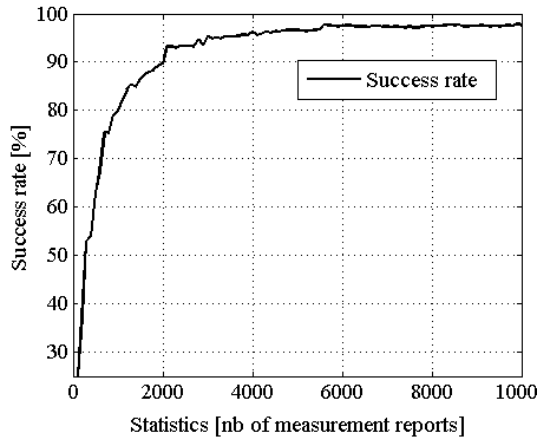

(c) convergence speed: $v=20 \mathrm{~m} / \mathrm{s}$

Fig. 5. Performance evaluation. Simulated with 20 serving cells.

flat performance and are not further improved because having limited knowledge about the neighbour cells coverage prevents the configuration methods from adding useful neighbour cells.

\section{Convergence Speed of the Self-Optimization Method}

A further important aspect that was evaluated is the convergence speed of the self-optimization method. This provides information on from which point the self-optimization method provides reliable performances that outperform the self-configuration method used for initialization. Fig. 5(c) shows the achievable performance for different numbers of reported measurements. It is shown that the more measurements are available, the better the quality of the self-optimized NCL is, since more reliable information about the neighbour cell coverage is available.

At about 3000 measurements, the self-optimization algorithm provides a success rate about $95 \%$. And at 6000 available measurements, the success rate attains $99 \%$. Since the selfconfiguration achieves an average performance of approximately $95 \%$ when the list size is sufficient large as shown in Fig. 5(b), it makes sense to switch to the NCL obtained from the self-optimization algorithm once more than approximately 3000 measurement are available. From this point on, the selfoptimized NCL provides improved performance.

\section{CONCLUSION}

A method for automatically optimizing a neighbour cell list was presented. The proposed approach consists of a selfconfiguration phase for initialization, followed by a switch to a self-optimization phase once sufficient measurements are reported by connected mobile stations. For initialization, the measurements-based self-configuration method achieved the best performance of approximately $95 \%$ in handover target searching success rate with 10 or more neighbour cells. The success rate is further improved by switching to a NCL obtained by the proposed self-optimization method. It was shown that a switch to a self-optimized NCL can improve the performance once more than approximately 3000 measurements reports are collected, and a near maximum performance of around 99\% success rate is achieved after more than around 6000 measurements reports are available. The proposed solutions are simple but efficient by using true measurementbased self-optimization algorithms. These suggest that the optimization of NCLs can be automated, which will help to reduce operational expenses in future cellular networks.

\section{ACKNOWLEDGMENT}

This work was partially supported by POSEIDON project within the French cluster Systemetic.

\section{REFERENCES}

[1] H. Olofsson, S. Magnusson, and M. Almgren, "A concept for dynamic neighbor cell list planning in a cellular system," in IEEE PIMRC '96, vol. 1, 1996, pp. 138-142.

[2] M. Amirijoo, P. Frenger, F. Gunnarsson, H. Kallin, J. Moe, and K. Zetterberg, "Neighbor cell relation list and physical cell identity self-organization in LTE,' in IEEE ICC '08 Workshops, May 2008, pp. $37-41$.

[3] NGMN Alliance, "Use cases related to self-organising network: Overall description,” Tech. Rep. v2.02, Dec. 2008.

[4] — "Recommendation on SON and O\&M requirements," Req. Spec. v1.23, Dec. 2008.

[5] S. Magnusson and H. Olofsson, "Dynamic neighbor cell list planning in a microcellular network," in IEEE 6th International Conference on Universal Personal Communications, Oct. 1997, pp. 223-227 vol.1.

[6] R. Guerzoni, I. Ore, K. Valkealahti, and D. Soldani, "Automatic Neighbor Cell List Optimization for UTRA FDD Networks: Theoretical Approach and Experimental Validation," in IWS/WPMC, Denmark, 2005.

[7] D. Soldani and I. Ore, "Self-optimizing neighbor cell list for UTRA FDD networks using detected set reporting," in IEEE VTC2007-Spring, 2007, pp. 694-698.

[8] F. Parodi, M. Kylvaja, G. Alford, J. Li, and J. Pradas, "An automatic procedure for neighbor cell list definition in cellular networks," in IEEE WoWMoM '07, June 2007, pp. 1-6.

[9] J. Li and R. Jantti, "On the study of self-configuration neighbour cell list for mobile WiMAX," in The 2007 International Conference on Next Generation Mobile Applications, Services and Technologies, NGMAST '07, Sept. 2007, pp. 199-204.

[10] M. Amirijoo, P. Frenger, F. Gunnarsson, H. Kallin, J. Moe, and K. Zetterberg, "Neighbor cell relation list and measured cell identity management in LTE," in IEEE Network Operations and Management Symposium, NOMS '08, Apr. 2008, pp. 152-159.

[11] 3GPP TS 25.133, "Requirements for support of Radio Resource Management FDD (Release 8)," Tech. Spec. v8.9.0, Dec. 2009.

[12] 3GPP TR 25.913, "Requirements for Evolved UTRA and Evolved UTRAN," Tech. Rep. v8.0.0, Dec. 2008.

[13] M. Zonoozi and P. Dassanayake, "User mobility modeling and characterization of mobility patterns," IEEE Journal on Selected Areas in Communications, vol. 15, no. 7, pp. 1239-1252, Sept. 1997.

[14] W. Wang and M. Zhao, "Joint effects of radio channels and node mobility on link dynamics in wireless networks," in IEEE INFOCOM '08, Apr. 2008, pp. 933-941. 